\title{
Ion transporter agonist improves recovery
}

Most spinal cord injuries (SCIs), even those that result in complete paralysis, are anatomically incomplete. Zhigang He and colleagues have now shown that agonists of $\mathrm{Na}^{+} / 2 \mathrm{Cl}^{-} / \mathrm{K}^{+}$ cotransporter 2 (KCC2) can reactivate the spared axons in mice, leading to functional recovery.

The authors used the staggered lesion model of SCI, in which two lateral hemisections are performed at the 7 th and 10th thoracic vertebrae (T7 and T10). In this procedure, which results in nearly complete and permanent hindlimb paralysis, only the axons that cross the midline between $\mathrm{T} 7$ and $\mathrm{T} 10$ remain intact. In this intra-lesion region, some descending axons that were spared in the T7 hemisection could, in theory, re-establish connections with those that were spared in the T10 section.

Using this model, the authors tested candidate compounds, selected based on bioavailability and the known roles of their targets in altering neuronal excitability, for their ability to mediate recovery. Among these, CLP290, a KCC2 agonist initially developed to treat neuropathic pain, was the only compound that resulted in substantial, long-term improvement in stepping ability. Although serotonergic agonists induced acute improvements, these improvements were transient. The effects of CLP290 first appeared after 4-5 weeks of daily treatment; $80 \%$ of CLP290-treated mice recovered stepping function. These improvements were maintained for 1-2 weeks after ending treatment.

The effects of CLP290 were limited to mice with incomplete lesions, and the compound did not affect axon regrowth.

Overexpression of KCC2 in neurons of the central nervous system in mice - specifically inhibitory interneurons in the region between the two lesions - mimicked the effects of CLP290 in promoting functional recovery. KCC2 agonists therefore likely mediate changes in the inhibitory neurons of the relay zone, between the two hemisections.

Immature neurons contain high levels of intracellular chloride. Thus, in these neurons, GABA and glycine, which open chloride channels, are depolarizing and excitatory. In early postnatal life the levels of intracellular chloride drop, and the effects of GABA and glycine become inhibitory. KCC2 upregulation occurs during the early postnatal period and contributes to this change in intracellular chloride levels. The authors therefore postulated that the KCC2 downregulation, which occurs following SCI, could result in inhibitory neurons adopting a more immature state in which classically inhibitory neurotransmitters are depolarizing. KCC2 agonists would restore a more physiological state, and inputs from descending neurons would have the intended effects. Indeed, using c-Fos staining as a proxy for neuronal activity, SCI decreased c-Fos staining in the intermediate and ventral spinal cord, and treatment with CLP290 or overexpression of KCC2 in these mice restored the expression pattern.

To confirm this change in excitability, the authors expressed a designer receptor exclusively activated by designer drugs (DREADD) coupled to inhibitory $G$ protein $\left(G_{i}\right)$ in inhibitory interneurons between and around the lesions. Upon activation of DREADD- $\mathrm{G}_{\mathrm{i}}$, injured mice recovered to a similar degree as those treated with CLP290 or those overexpressing KCC2. Thus, reducing the excitability of excitatory interneurons can increase the responsiveness to descending inputs and promote recovery after SCI.

He explains, "In contrast to other compounds we tested, CLP290 treatment, even at high doses, has no obvious side effects." He also highlights that "CLP290 is not a typical agonist, because it was shown to move KCC2 from intracellular compartments to the cell surface," which is an unusual mechanism. Furthermore, "it is unclear whether CLP290 binds directly to KCC2," he says.

CLP290 was discovered and developed by the now-defunct Chlorion Pharma. Although further work needs to be done before this molecule could be brought to the clinic, therapies that reduce the excitability of excitatory interneurons might be useful in patients with SCIs.

Megan Cully

ORIGINAL ARTICLE Chen, B. et al. Reactivation of dormant relay pathways in injured spinal cord by KCC2 manipulations. Cell https://doi. org/10.1016/j.cell.2018.06.005 (2018) 